S. Gallego et al.

Personality, Peers and Alcohol Use in Adolescents

\title{
Contribution of the Five Factors of Personality and Peers on Adolescent Alcohol Use: A Cross-National Study
}

Sígrid Gallego ${ }^{1}$, Laura Mezquita ${ }^{1}$, Jorge Moya-Higueras ${ }^{2}$, Generós Ortet ${ }^{1,3}$, and Manuel I. Ibáñez ${ }^{1}$

\section{${ }^{1}$ Universitat Jaume I (Spain) \\ ${ }^{2}$ Universitat de Lleida (Spain)}

${ }^{3}$ Centro de Investigación Biomédica en Red de Salud Mental (Spain)

Ministerio de Economía y Competitividad/FEDER, PSI2015-67766-R. Generalitat Valenciana, GV/2016/158

Correspondence concerning this article should be addressed to Generós Ortet. Universitat Jaume I. Departament de Psicologia Bàsica, Clínica i Psicobiologia. 12071 Castelló de la Plana (Spain). E-mail: ortet@uji.es

\section{How to cite this article:}

Gallego, S., Mezquita, L., Moya-Higueras, J., Ortet, G., \& Ibáñez, M. I. (2018). Contribution of the five factors of personality and peers on adolescent alcohol use: A cross-national study.

The Spanish Journal of Psychology, 21. e58. https://doi.org/10.1017/sjp.2018.63 


\begin{abstract}
Personality characteristics such as extraversion, low agreeableness and low conscientiousness are relevant for alcohol use during adolescence. In addition, having friends who use alcohol is one of the strongest predictors of adolescent alcohol consumption and its negative outcomes. The selection model posits that friends display similar alcohol consumption when their friendships are formed on the basis of common characteristics as, among others, personality. Thus, the aim of this study was to examine the mediation role of peers in the association between the five-factor model of personality and adolescent alcohol use in two cultures. One hundred and twenty Scottish and 221 Spanish respondents, all aged 12-15 years, answered the Alcohol Intake Scale (AIS). Adolescents were asked about the alcohol used at the weekend and also about the alcohol consumed by their friends. Scottish adolescents' personality was measured by the NEO Personality Inventory-3 (NEO-PI-3). The Junior Spanish version of the Revised NEO Personality Inventory (JS NEO) was used to assess personality in the Spanish sample. Low agreeableness and low conscientiousness correlated with own alcohol quantity in both countries. We performed an independent structural equation modeling for each country. Extraversion $(\beta=.205, p<.05)$ and low agreeableness $(\beta=-.196, p<.01)$ for Scottish adolescents, and low conscientiousness $(\beta=-$ $.175, p<.05)$ for Spanish youths, predicted alcohol use through peer alcohol consumption at weekends. These findings support the relevance of personality traits and peer affiliation in relation to alcohol consumption in adolescence.
\end{abstract}

Received 21 March 2018; Revised 5 November 2018; Accepted 6 November 2018

Keywords: adolescent alcohol consumption, cross-national, Five-Factor Model, peers, personality. 
Alcohol use initiates and binge drinking escalates in many young people aged 13-15 years (Windle, 2016). A study from 35 European countries indicates that nearly half of adolescents (47\%) report alcohol use, and one in twelve have experienced intoxication by the age of 13 or younger (European School Survey Project on Alcohol and Other Drugs ESPAD, 2016). For instance, Spanish high school students report starting their alcohol consumption at 13.9 years and $76.8 \%$ of them have consumed alcohol during the last year, while $50.1 \%$ have drunk at least once (Delegación del Gobierno para el Plan Nacional sobre Drogas DGPNSD, 2016). Among Scottish teenagers, less than a third of 13-year-olds (28\%) and two thirds of 15 -year-olds (66\%) have had an alcoholic drink. Around two thirds of the 15-year-olds (68\%) who have drunk alcohol have been drunk at least once; the average age that 15-year-olds first drink is 13 years and 2 months (The Scottish Government, 2016). Thus the understanding of the risk factors associated with alcohol use in adolescence has important implications for the development of more effective prevention and intervention programs for alcohol abuse and alcoholism (Golpe, Isorna, Barreiro, Braña, \& Rial, 2017).

Peer groups form an important interpersonal and social context in adolescence, especially when predicting alcohol use (Ciairano, Bosma, Miceli, \& Settanni, 2008; Espejo, Cortés, Martín del Río, Giménez, \& Gómez, 2012; Knecht, Weesie, Burk, \& Steglich, 2010). Studies have consistently found that affiliation with friends who use alcohol is one of the strongest predictors of adolescent alcohol consumption and its negative consequences (Dick et al., 2013; Grigsby, Forster, Unger, \& Sussman, 2016; Harden, Hill, Turkheimer, \& Emery, 2008). Bauman and Ennett (1996) proposed that the association between friends and adolescent alcohol use could be explained by influence and selection models. The influence model posits that friendships cause alcohol consumption. Accordingly, the peer environment may contribute to alcohol use by means of direct influences, modeling, availability and acceptance of drinking behaviors, among others (Knecht et al., 2010). However, adolescents do not randomly choose their friends, rather certain factors, such as attitudes, personality and drinking behavior, will increase the likelihood of an adolescent choosing a best friend(s) who use(s) alcohol (Harden et al., 2008). Thus the selection model posits that friends display similar alcohol consumption when their friendships are formed on the basis of common alcohol use. This means that adolescents select peers based on their own drinking behaviors (Edwards, Maes, Prescott, \& Kendler, 2015). Cross-sectional, longitudinal and genetically informative studies have found that friendship selection plays a relatively stronger role than peer influence when explaining the similarity of adolescent friends' alcohol use (Dick et al., 2013; Edwards et al., 2015; Knecht et al., 2010). However once friendships are formed, they 
may reinforce common behaviors, in which case they operate as an influence among alcohol users (Osgood et al., 2013).

When considering the psychological variables related to alcohol consumption, personality traits are relevant factors to predict alcohol use and abuse (Grigsby et al., 2016; Hakulinen, et al., 2015; Kotov, Gamez, Schmidt, \& Watson, 2010). Trait psychology models assume that a number of consistent relatively stable traits are the core of personality, which have a moderate biological and genetic influence, and interact with environmental factors (Boyle, Matthews, \& Saklofske, 2008). The Five-Factor Model (FFM) of personality offers a useful descriptive taxonomy for most personality traits according to many personality psychologists (McCrae \& Costa, 2010). The FFM proposes the broad traits of neuroticism, extraversion, openness to experience, agreeableness and conscientiousness. Neuroticism refers to individual differences in the tendency to experience negative emotions frequently and intensively, such as anxiety, fear, depression, irritability and having low self-esteem; extraversion reflects individual differences in sociability, social ascendency, activity, excitement seeking and positive emotionality; openness to experience represents individual differences in curiosity, fantasy, appreciation of art and beauty, and social attitudes; agreeableness reveals individual differences in compliance, empathy, collaboration and altruism; conscientiousness represents individual differences in being methodical, planning, impulse control, and also to respecting and abiding by conventional social norms and rules (Morizot, 2014). Most previous studies on alcohol use and alcoholism within the FFM framework have been carried out in adults. The results indicate that low conscientiousness and low agreeableness are associated with both regular and problematic alcohol consumption (Hakulinen, et al., 2015; Malouff, Thorsteinsson, Rooke, \& Schutte, 2007). In their metaanalysis that included 175 studies, Kotov et al. (2010) have also found that substance use disorders present low conscientiousness, but agreeableness is unrelated mostly to this diagnosis. Regarding extraversion, Mezquita, Ibáñez, Moya, Villa, and Ortet (2014) have found, in a five-year longitudinal study, that a positive affect regulation pathway is associated with more recreational alcohol use, in which this dimension plays a prominent role. Hakulinen et al. (2015) have shown, in a meta-analysis using data from eight cohort studies, that high alcohol consumption is commoner among extraverts. Moreover, extraversion seems more relevant in adolescent alcohol use that in adults, although FFM domains have been seldom explored at these ages (Dick et al., 2013; Ibáñez et al., 2015). Regarding neuroticism, it has been proposed that alcohol use disorders may develop because alcohol relieves the negative affect. So this broad dimension may play a significant role in problematic alcohol 
consumption through negative affect regulation (Kotov et al., 2010; Malouff et al., 2007; Mezquita et al., 2014). Finally, openness to experience has been less intensely explored in relation to alcohol consumption. For example, Hakulinen et al. (2015) have found that low openness to experience may increase the odds of reducing alcohol consumption and preferring abstinence in adults, although other studies suggest a negligible role of this personality domain in this behavior (Kotov et al., 2010).

The peer selection model indicates that friendships are formed on the bases of common alcohol consumption, but also personality (Edwards et al., 2015). According to the studies reviewed above, both friend selection and personality are relevant factors of alcohol use in adolescence. Very few studies have investigated personality and alcohol use within the FFM in adolescents (Ibáñez et al., 2015), and studies of adolescent alcohol use that have compared adolescents across countries are scarce (Ciairano et al., 2008). Due to cultural factors (Room, 2001), Spanish and Scottish adolescents have been hypothesized to present different drinking behaviors. Scottish people would live in a "dry" alcohol culture, characterized by drinking at certain times, such as weekends, to become intoxicated. On the contrary, the Spanish drinking culture is considered "wet", described as a consumption that is integrated into daily life or Mediterranean diet, but with low to moderate quantities. However, a cross-national study (Soellner, Göbel, Scheithauer, \& Bräker, 2014) has shown that this classical difference is not as clear as it used to be, and more similarities than differences for alcohol consumption habits across Europe have been found. One of the most common similarities among countries is that adolescents drink alcohol with friends at the weekend (DGPNSD, 2016). One possible explanation for this homogenization is that the same risk factors (personality and peers' alcohol use, among others) could operate across countries. Hence the present cross-national research appears necessary to replicate the contribution of factors like peers and personality traits on alcohol consumption in different countries (Bauman \& Ennett, 1996; Ciairano et al., 2008; Osgood et al., 2013).

The main aim of the present research was to study the contribution of FFM personality traits and friend alcohol consumption on adolescents' own alcohol use. We hypothesized that adolescents with higher scores for the personality traits associated with alcohol use would choose friends who consume more alcohol at weekends, which is when most adolescents drink with friends. Accordingly, low conscientiousness and low agreeableness and, to a lesser degree, high extraversion would be related to peer alcohol consumption that, in turn, would influence own alcohol use. The model tested here is shown in Figure 1. As the study was carried out in two different countries, we also hypothesized that the predicted relationships 
would be the same in both cultures.

\section{Method}

\section{Participants and procedure}

The study was carried out in public high schools in Scotland and Spain, and recruitment of participants was not standardized. All the attending students voluntarily completed the questionnaires in the selected classrooms and did not receive any compensation for their participation. The Glasgow City Council and the Ethics Committee of the Universitat Jaume I both approved the present study.

When we analyzed the distribution of the quantity of alcohol used, two participants, one Scottish and one Spanish, indicated a number of drinks more than four standard deviations over the mean for each country. So, they were identified as clear outliers and excluded from the data set. The Scottish sample consisted of 120 high school students (62 females, 58 males, $M_{\text {age }}=13.68$ years, age range: $12-15$ years) who answered the NEO-PI-3 (McCrae \& Costa, 2010). The Spanish sample comprised 221 adolescents (121 females, 100 males, $M_{\text {age }}=13.53$ years, age range: $12-15$ years) who completed the Junior version of the Spanish NEO-PI-R (JS NEO; Ortet et al., 2012). Both Scottish and Spanish student samples answered the Alcohol Intake Scale (AIS; Ibáñez et al., 2015).

\section{Measures}

Personality. The Scottish students answered the NEO-PI-3 (McCrae \& Costa, 2010). It comprises 240 items that are answered on a 5-point Likert scale ranging from strongly disagree to strongly agree. It assesses 30 specific traits or facets, from 12 years and upwards, that define the FFM personality factors or domains: Neuroticism, extraversion, openness to experience, agreeableness and conscientiousness. Each domain score is obtained by aggregating scores on their six facets, and each personality facet has eight items. The psychometric characteristics of the questionnaire are presented in the manual (McCrae \& Costa, 2010). In our Scottish sample, Cronbach's alphas were .85 for neuroticism, .85 for extraversion, .77 for openness, .85 for agreeableness, and .88 for conscientiousness.

The Spanish sample completed the Junior version of the Spanish NEO-PI-R (JS NEO; Ortet et al., 2012). This inventory also includes 240 items ( 8 items per facet x 30 facets) and assesses the same FFM dimensions in adolescents aged 12-17 years. Its psychometric characteristics are shown in Ortet et al. (2012). Alpha reliabilities are .88, .85, .82, .82, and .91 for neuroticism, extraversion, openness, agreeableness, and conscientiousness respectively. 
Alcohol consumption. The Alcohol Intake Scale (AIS; Ibáñez et al., 2015) was used to assess alcohol use in both student samples. Adolescents were asked to indicate the frequency with which they had drunk the following beverages: Beer, wine, liquors and mixed drinks. The answer categories were: Never or almost never, 1-3 times per month, 1-6 times per week and daily. They also indicated the amount of Standard Drink Units (SDUs) drunk at the weekend, when was most likely to drink with friends, by taking into account that beers and glasses of wine are the equivalent to one SDU. Liquors and mixed drinks are the equivalent to one SDU in the United Kingdom (UK) (Department of Health, 2016; National Health Service, 2015), and to two SDUs in Spain (Ministerio de Salud, Servicios Sociales e Igualdad, 2016). The total number of SDUs consumed (summed) were transformed into grams of alcohol. One SDU is the equivalent to 8 and 10 grams of alcohol in the UK and Spain, respectively (International Alliance for Responsible Drinking, 2016; Mongan \& Long, 2015). Finally, students also indicated how much of the above listed alcoholic beverages they thought their best friend and group of friends drank at the weekend. The response options were: nothing or almost nothing, a little, quite a lot and a lot. Finally, our data (see Table 1) are in accordance with the alcohol statistics in both countries (DGPNSD, 2016; The Scottish Government, 2016).

\section{Data analysis}

We intended to know whether personality influenced alcohol use through peers' alcohol consumption, and if these associations were similar in different cultures. Thus, a structural equation model (SEM), in which the personality dimensions were the independent variables, peers' alcohol use was the mediator and adolescent alcohol use was the dependent variable, was carried out independently with each sample. A different questionnaire was used to measure the FFM personality traits in each country, so their scores could not be considered together and a multi-group SEM could not be performed to test moderation between countries. Robust methods were used given the non-normality in the data. Structural equations were performed with the EQS 6.2 SEM software.

The used goodness-of-fit indices were: the s-B $\chi^{2}$ test, the NFI, CFI, IFI, MFI, RMSEA and $90 \%$ CI of RMSEA. $p$ values higher than .05 of the $\chi^{2}$ test should indicate that the model fitted the data. Values higher than .95 for NFI, CFI, MFI and lower than .06 for RMSEA were considered representative of an adequate model fit (Byrne, 2006).

\section{Results}

\section{Descriptive statistics}


The means and standard deviations of the frequency and amount of alcohol consumed in grams at weekends, and the best friend and group of friends' alcohol use also at the weekend, are shown in Table 1. The comparison of the alcohol-related variables between Scotland and Spain indicated a significantly higher consumption among Scottish youths in own alcohol frequency, best friend and group of friends' alcohol use on weekends. There were not significant differences between the two countries in the quantity consumed at the weekend. Scottish and Spanish adolescents consumed the equivalent of about one and a half beers per weekend.

\section{Tabla 1 aproximadamente aquí}

\section{Preliminary analysis to SEM}

Table 2 shows the correlations among the personality scales and the alcohol-related variables. The usual negative correlations between neuroticism and conscientiousness, and the positive correlations between extraversion and openness to experience, were also found in both the NEO-PI-3 and the JS NEO. However, agreeableness and conscientiousness were also positively associated in Scotland and Spain, and openness to experience correlated with agreeableness and conscientiousness in both questionnaires. Finally, there was a significant negative correlation between neuroticism and extraversion in the NEO-PI-3. Regarding the correlations between the personality dimensions and the alcohol-related scales, agreeableness inversely correlated to own and best friend alcohol consumptions in Scotland; and to own, best friend and group of friends in Spain. Low conscientiousness also related to adolescent alcohol use, but all the correlations were significant only in the Spanish sample. Extraversion showed low correlations for most variables in both countries. Neuroticism and openness to experience did not relate to alcohol use in either sample.

\section{SEM}

We tested the hypothesized model (see Figures 1 and 2) independently in each sample. Both s-B $\chi^{2}$ were nonsignificant and all the rest of fit indices were adequate for both countries, with the exception of the RMSEA in the Scottish sample, which was higher than .05 (see Table 3). All the indirect and total effects are presented in Table 4. The indices in Figure 2 and Table 4 show that the effect of extraversion on adolescent alcohol use was totally mediated by peers' alcohol use at weekends, while (low) agreeableness had both indirect and direct effects in the Scottish adolescents. In the Spanish sample, there were no direct effects between personality dimensions and adolescent drinking. However, the dimension that influenced own consumption through peers' alcohol use at the weekends was (low) conscientiousness 


\section{Discussion}

The main goal of the present study was to cross-nationally test whether the FFM personality traits influenced adolescent own alcohol use through peer alcohol consumption on weekends. The SEM showed that low agreeableness and high extraversion were associated with adolescent alcohol use through friends' alcohol consumption in Scotland. However, in Spain, the personality dimension that influenced own drinking through friends' alcohol use at the weekend was (low) conscientiousness. Thus, as expected, personality dimensions were related to adolescent alcohol use through friends drinking. However, contrary to our predictions, the personality characteristics that influenced alcohol consumption with friends were different for each country.

Although we found a significant higher consumption in Scotland than in Spain for alcohol frequency (but not quantity) and friends alcohol use, the present study replicated the finding that peers' alcohol use is one of the main factors that influences adolescent drinking behavior at weekends. This risk factor could partially explain the trend toward the homogenization of drinking patterns that takes place across different countries in Europe (Soellner et al., 2014). Moreover, personality traits are also relevant for alcohol use, although most studies under the FFM framework have been carried out in adults (Hakulinen et al., 2015). Our findings add further evidence that extraversion, (low) conscientiousness and (low) agreeableness are also related to alcohol consumption in adolescents, mainly through peers's alcohol use. According to the friends' selection model (Bauman \& Ennett, 1996; Dick et al., 2013; Edwards et al., 2015), those adolescents with a personality profile associated with alcohol use (especially extraverted and antagonistic in Scotland, and unconscientious in Spain) would choose friends who drink more alcohol.

Different FFM personality domains would be associated with alcohol use and abuse through distinct pathways. Thus, Mezquita et al. (2014) showed that the association between low agreeableness and low conscientiousness and alcohol regular use and alcohol-related problems were mediated by antisocial behavior, supporting the existence of a deviance proneness pathway that predicted alcohol outcomes in young adults. Our results also showed the importance of low agreeableness on both, own alcohol use and the affiliation with friends who consume alcohol, which supports the importance of a deviance proneness pathway in adolescence, especially in Scotland. This result may reflect a tougher control for underage drinking in Scotland, which would be perceived as more antisocial (breaking the law) than in Spain. We also found that low conscientiousness (i.e., impulsivity-related traits such as 
sensation seeking or low premeditation) was relevant for alcohol consumption mainly in Spanish adolescents. The role of impulsivity-related traits on adolescent alcohol use has been well established (Stautz \& Cooper, 2013), and our results suggest that this association may be due, at least in part, to the selection of peers who consume alcohol.

Finally, our results also showed that extraversion was related to alcohol use. We found a significant indirect effect (through peers) of extraversion on own drinking in Scotland, and a tendency for the total effects on own drinking in both countries (see Table 4). It would seem that this dimension played a more important role in adolescent alcohol consumption than in adults (Ibáñez et al., 2015), especially through joining friends who drink alcohol. Accordingly, our finding of total mediation through peers' alcohol use in Scotland could indicate that extraversion was associated with social recreational alcohol use, thus extraverted youths would drink to find more opportunities for social relations because opportunities for meeting other people are very often opportunities for drinking alcohol and for moodenhancement (Fairbairn et al., 2015).

Cross-national studies that have examined peers and personality factors in relation to adolescent alcohol use are almost nonexistent. The need to and relevance of conducting the study of psychological variables in diverse cultural groups have been emphasized (Ciairano et al., 2008; Knecht et al., 2010; Osgood et al., 2013). As far as we know, this is the first work to provide empirical evidence for the interplay of the FFM personality traits and friends' consumption in adolescent alcohol use across different countries. This contribution could help to improve the efficiency of prevention and intervention programs by targeting alcohol use in adolescents.

This study presented three main limitations. First, a different FFM personality inventory for adolescents was used in each country. It was not possible to use the NEO-PI-3 in Spain when we administered the questionnaire as the Spanish language adaptation of the inventory is still underway. Similarly, the JS NEO is available only in Spanish. Nonetheless, both instruments derive from the original NEO-PI-R (McCrae \& Costa, 2010) and assess the same personality constructs. Second, friends' alcohol consumption was measured by asking adolescents to describe their peer alcohol use. It would be more appropriate if friends reported their actual alcohol use as relationships between friends and adolescent alcohol use tend to be less strongly related when friends' own drinking is taken into account (Bauman \& Ennett, 1996; Edwards et al., 2015). Third, this study was cross-sectional, so our results cannot provide clear evidence on the selection or the alternative influence models. Furthermore, although not likely present in our data, other studies have proposed the role of the reverse 
causality in the relation between personality and alcohol use over time (i.e., alcohol use may be related to personality trait changes). For instance, Hakulinen and Jokela (2018) found that risky alcohol use was associated with increasing extraversion and decreasing emotional stability, agreeableness, and conscientiousness in adults. Thus, prospective longitudinal designs are needed to disentangle the specific processes that underlie the association between personality, peers' drinking behavior and adolescent alcohol use.

Despite these limitations, we conclude that personality traits may influence adolescents' alcohol consumption through peer alcohol use across countries. Antagonistic and extraverted Scottish adolescents, and unconscientious Spanish youths, may tend to select a peer network that drinks more at weekends and, in turn, the group of friends' alcohol consumption, especially at the weekend, facilitates the availability and use of own alcoholic beverages. 


\section{References}

Bauman, K. E., \& Ennett, S. T. (1996). On the importance of peer influence for adolescent drug use: Commonly neglected considerations. Addiction, 91, 185-198. http://dx.doi.org/10.1046/j.1360-0443.1996.9121852.x

Boyle, G. J., Matthews, G., \& Saklofske, D. H. (2008). Personality theories and models: An overview. In G. J. Boyle, G. Matthews, \& D. H. Saklofske (Eds.), Personality theory and assessment. Personality theories and models (Vol. 1, pp. 1-29). London, UK: Sage.

Byrne, B. M. (2006). Structural equation modeling with EQS: Basic concepts, applications, and programming. Mahwah, NJ: Lawrence Erlbaum.

Ciairano, S., Bosma, H. A., Miceli, R., \& Settanni, M. (2008). Adolescent substance use in two European countries: Relationships with psychosocial adjustment, peers, and activities. International Journal of Clinical and Health Psychology, 8, 119-138.

Cohen, J. (1992). A power primer. Psychological Bulletin, 112, 155-159. http://dx.doi.org/10.1037/0033-2909.112.1.155

Espejo, B., Cortés, M. T., Martín del Río, B., Giménez, J. A., \& Gómez, C. (2012). Traits that define the different alcohol intensive consume type during the practice of "botellón". The Spanish Journal of Psychology, 15, 256-264. https://doi.org/10.5209/rev_SJOP.2012.v15.n1.37318

Department of Health (2016). UK Chief Medical Officers' Low Risk Drinking Guidelines. Retrieved from https://www.gov.uk/government/uploads/system/uploads/attachment data/file/545937/UK C MOs report.pdf

Delegación del Gobierno para el Plan Nacional sobre Drogas (DGPNSD) (2016). Informe de la encuesta estatal sobre uso de drogas en estudiantes de enseñanzas secundarias (estudes) 2014/15 [Report of the national survey on drug use in high school students (estudes) 2014/15]. Retrieved from https://www.fundadeps.org/actividades/documentos/655/2016_ESTUDES_2014-2015.pdf

Dick, D. M., Aliev, F., Latendresse, S. J., Hickman, M., Heron, J., Macleod, J., ... Kendler, K. S. (2013). Adolescent alcohol use is predicted by childhood temperament factors before age 5, with mediation through personality and peers. Alcoholism: Clinical and Experimental Research, 37, 218-2117. http://dx.doi.org/10.1111/acer.12206

Edwards, A. C., Maes, H. H., Prescott, C. A., \& Kendler, K. S. (2015). Multiple mechanisms influencing the relationship between alcohol consumption and peer alcohol use. Alcoholism: Clinical and Experimental Research, 39, 324-332. http://dx.doi.org/10.1111/acer.12624

European School Survey Project on Alcohol and Other Drugs (ESPAD) (2016). ESPAD Report 2015. Results from the European School Survey Project on Alcohol and Other Drugs. Retrieved from http://www.espad.org/sites/espad.org/files/ESPAD report 2015.pdf

Fairbairn, C. E., Sayette, M. A., Wright, A. G. C., Levine, J. M., Cohn, J. F., \& Creswell, K. G. (2015). Extraversion and the rewarding effects of alcohol in a social context. Journal of Abnormal Psychology, 124, 660-673. http://doi.org/10.1037/abn0000024

Golpe, S., Isorna, M., Barreiro, C., Braña, T., \& Rial, A. (2017). Binge drinking among adolescents: Prevalence, risk practices and related variables. Adicciones, 29, 256-267.

Grigsby, T. J., Forster, M., Unger, J. B., \& Sussman, S. (2016). Predictors of alcohol-related negative consequences in adolescents: A systematic review of the literature and implications for future research. Journal of Adolescence, 48, 18-35. http://dx.doi.org/10.1016/j.adolescence.2016.01.006

Hakulinen, C., \& Jokela, M. (2018). Alcohol use and personality trait change: Pooled analysis of six cohort studies. Psychological Medicine, 1-8. https://doi.org/10.1017/S0033291718000636

Hakulinen, C., Elovainio, M., Batty, G. D., Virtanen, M., Kivimäki, M., \& Jokela, M. (2015). Personality and alcohol consumption: Pooled analysis of 72,949 adults from eight cohort studies. Drug Alcohol Dependence, 151, 110-114. http://doi.org/10.1016/j.drugalcdep.2015.03.008

Harden, K. P., Hill, J. E., Turkheimer, E., \& Emery, R. E. (2008). Gene-environment correlation and interaction in peer effects on adolescent alcohol and tobacco use. Behavior Genetics, 38, 339- 
347. http://dx.doi.org/10.1007/s10519-008-9202-7

Ibáñez, M. I., Camacho, L., Mezquita, L., Villa, H., Moya-Higueras, J., \& Ortet, G. (2015). Alcohol expectancies mediate and moderate the associations between Big Five personality traits and adolescent alcohol consumption and alcohol-related problems. Frontiers in Psychology, 6. http://doi.org/10.3389/fpsyg.2015.01838

International Alliance for Responsible Drinking (2016). Drinking guidelines: General population. Retrieved from http://www.iard.org/policy-tables/drinking-guidelines-general-population

Knecht, A. B., Weesie, J., Burk, W. J., \& Steglich, C. (2010). Friendship and alcohol use in early adolescence: A multilevel social network approach. Journal of Research on Adolescence, 21, 475-487. https://doi.org/10.1111/j.1532-7795.2010.00685.x

Kotov, R., Gamez, W., Schmidt, F., \& Watson, D. (2010). Linking "big" personality traits to anxiety, depressive, and substance use disorders: A meta-analysis. Psychological Bulletin, 136, 768821. http://psycnet.apa.org/doi/10.1037/a0020327

Malouff, J. M., Thorsteinsson, E. B., Rooke, S. E., \& Schutte, N. S. (2007). Alcohol involvement and the Five-Factor Model of personality: A meta-analysis. Journal of Drug Education, 37, $277-$ 294.

McCrae, R. R., \& Costa, P. T. (2010). NEO Inventories for the NEO Personality Inventory-3 (NEO$P I-3), N E O$ Five-Factor Inventory-3 (NEO-FFI-3), NEO Personality Inventory-Revised (NEO-PI-R): Professional manual. Lutz, FL: Psychological Assessment Resources.

Mezquita, L., Ibáñez, M. I., Moya, J., Villa, H., \& Ortet, G. (2014). A longitudinal examination of different etiological pathways to alcohol use and misuse. Alcoholism: Clinical and Experimental Research, 38, 1770-1779. http://doi.org/10.1111/acer.12419

Ministerio de Salud, Servicios Sociales e Igualdad Healthy lifestyles: Risky and harmful alcohol consumption. Retrieved from http://www.estilosdevidasaludable.msssi.gob.es/en/consumo/queSaber/home.htm

Mongan, D., \& Long, J. (2015). Standard drink measures throughout Europe. People's understanding of standard drinks and their use in drinking guidelines, alcohol survey and labeling. Retrieved from Reducing Alcohol Related Harm website:

http://www.rarha.eu/Resources/Deliverables/Lists/Deliverables/Attachments/14/WP5

Background paper Standard drink measures HRB.pdf

Morizot, J. (2014). Construct validity of adolescents' self-reported Big Five personality traits: Importance of conceptual breadth and initial validation of a short measure. Assessment, 21, 580-606. http://doi.org/10.1177/1073191114524015

National Health Service (2015). Alcohol misuse. Retrieved from https://www.nhs.uk/conditions/alcohol-misuse/

Ortet, G., Ibáñez, M. I., Moya, J., Villa, H., Viruela, A., \& Mezquita, L. (2012). Assessing the five factors of personality in adolescents: The junior version of the Spanish NEO-PI-R. Assessment, 19, 114-130. http://doi.org/10.1177/1073191111410166

Osgood, D. W., Ragan, D. T., Wallace, L., Gest, S. D., Feinberg, M. E., \& Moody, J. (2013). Peers and the emergence of alcohol use: Influence and selection processes in adolescent friendship networks. Journal of Research on Adolescence, 23, 500-512.

http://dx.doi.org/10.1111/jora.12059

Room, R. (2001). Intoxication and bad behavior: Understanding cultural differences in the link. Social Science \& Medicine, 53, 189-198. https://doi.org/10.1016/S0277-9536(00)00330-0

The Scottish Government (2016). Scottish Schools Adolescent Lifestyle and Substance Use Survey (SALSUS). Alcohol Report (2015). Retrieved from http://www.gov.scot/Resource/0050/00508470.pdf

Soellner, R., Göbel, K., Scheithauer, H., Bräker, A.-B. (2014). Alcohol use of adolescents from 25 European countries. Journal of Public Health, 22, 57-65. http://doi.org/10.1007/s10389-0130593-4

Stautz, K., \& Cooper, A. (2013). Impulsivity-related personality traits and adolescent alcohol use: A meta-analytic review. Clinical Psychology Review, 33, 574-592. http://doi.org/10.1016/j.cpr.2013.03.003

Windle, M., (2016). Drinking over the lifespan: Focus on early adolescents and youth. Alcohol Research: Current Reviews, 38, 95-101. 
Table 1.

Means and Standard Deviations for Personality Dimensions, Alcohol-Related Scales and Mean Comparison of Alcohol Consumption between Scottish and Spanish Adolescents.

\begin{tabular}{|c|c|c|c|c|c|c|}
\hline & \multicolumn{2}{|c|}{$\begin{array}{l}\text { Scotland } \\
(n=120)\end{array}$} & \multicolumn{2}{|c|}{$\begin{array}{c}\text { Spain } \\
(n=221)\end{array}$} & \multirow[b]{2}{*}{ t-test } & \multirow[b]{2}{*}{$d$} \\
\hline & $M$ & $S D$ & $M$ & $S D$ & & \\
\hline Neuroticism & 92.64 & 17.25 & 80.48 & 13.67 & & \\
\hline Extraversion & 116.68 & 16.57 & 96.17 & 12.48 & & \\
\hline Openness & 103.17 & 13.33 & 87.56 & 13.82 & & \\
\hline Agreeableness & 105.57 & 16.69 & 96.23 & 14.10 & & \\
\hline Conscientiousness & 102.47 & 18.81 & 96.83 & 15.02 & & \\
\hline Alcohol frequency & 1.30 & .42 & 1.12 & .25 & $4.44^{*}$ & .52 \\
\hline Alcohol quantity weekend & 23.88 & 41.74 & 15.65 & 37.03 & 1.87 & .21 \\
\hline Best friend alcohol use weekend & 1.53 & .70 & 1.21 & .46 & $4.58 *$ & .54 \\
\hline Group of friends' alcohol use weekend & 1.84 & .91 & 1.42 & .67 & $4.46^{*}$ & .53 \\
\hline
\end{tabular}

Note. Cohen's $d$ values of $.20, .50$, and .80 correspond to small, medium, and large effect sizes, respectively (Cohen, 1992).

$* p<.001$. 
Table 2.

Intercorrelations between Personality Dimensions and Alcohol-Related Variables.

\begin{tabular}{lccccccccc}
\hline & 1 & 2 & 3 & 4 & 5 & 6 & 7 & 8 & 9 \\
\hline 1. Neuroticism & - & -.08 & .07 & -.11 & $-.27^{* * *}$ & .04 & .09 & .01 & .11 \\
2. Extraversion & $-.44^{* * *}$ & - & $.31^{* *}$ & -.00 & .05 & .09 & .13 & .07 & .07 \\
3. Openness to experience & -.17 & $.36^{* * *}$ & - & $.23^{* * *}$ & $.30^{* * *}$ & -.10 & -.07 & -.09 & -.02 \\
4. Agreeableness & -.16 & .11 & $.37^{* * *}$ & - & $.36^{* * *}$ & $-.32^{* * *}$ & $-.27^{* * *}$ & $-.28^{* * *}$ & $-.18^{* *}$ \\
5. Conscientiousness & $-.49^{* * *}$ & $.25^{* *}$ & $.33^{* * *}$ & $.41^{* * *}$ & - & $-.25^{* * *}$ & $-.21^{* *}$ & $-.22^{* * *}$ & $-.24^{* * *}$ \\
6. Alcohol frequency & -.05 & .14 & -.06 & $-.26^{* *}$ & -.17 & - & $.63^{* * *}$ & $.59^{* * *}$ & $.50^{* * *}$ \\
7. Alcohol quantity weekend & .09 & .14 & -.07 & $-.29^{* *}$ & $-.21^{*}$ & $.36^{* * *}$ & - & $.62^{* * *}$ & $.52^{* * *}$ \\
8. Best friend alcohol use weekend & -.07 & $.19^{*}$ & -.15 & $-.19^{*}$ & -.09 & $.58^{* * *}$ & $.35^{* * *}$ & - & $.60^{* * *}$ \\
9. Group of friends' alcohol use weekend & .06 & $.25^{* *}$ & -.07 & -.17 & $-.24^{* *}$ & $.34^{* * *}$ & $.34^{* * *}$ & $.75^{* * *}$ & - \\
\hline
\end{tabular}

Note. Correlation coefficients below the diagonal are for Scottish adolescents; those above the diagonal are for Spanish adolescents. ${ }^{*} p<.05 .{ }^{* *} p<.01 .{ }^{* * *} p<.001$. 
Table 3.

Goodness-of-Fit Statistics of the Hypothesized Model.

\begin{tabular}{lcccccccc}
\hline & DF & S-B $\chi^{2}$ & $p$ & NFI & CFI & IFI & MFI & $\begin{array}{c}\text { RMSEA } \\
\text { [90\% CI RMSEA] }\end{array}$ \\
\hline Scottish adolescents & 8 & 15.208 & .055 & .945 & .970 & .973 & .970 & $.087[.000, .152]$ \\
Spanish adolescents & 8 & 8.503 & .386 & .975 & .998 & .998 & .999 & $.017[.000, .082]$ \\
\hline
\end{tabular}

Note. s-в $\chi^{2}=$ Satorra-Bendler chi-square; NFI = normed fit index; CFI = Bollen's fit index; IFI = incremental fit index; MFI = McDonald's fit index; RMSEA = root mean-square error of approximation. 
Table 4.

Indirect and Total Effects of the Structural Equation Models

\begin{tabular}{lcc}
\hline & $\begin{array}{c}\text { St. beta } \\
\text { Scottish } \\
\text { adolescents }\end{array}$ & $\begin{array}{c}\text { St. beta } \\
\text { Spanish } \\
\text { adolescents }\end{array}$ \\
\hline $\begin{array}{l}\text { Indirect effects } \\
\text { Extraversion } \rightarrow \text { Adolescent alcohol use }\end{array}$ & $.205^{*}$ & .085 \\
Agreeableness $\rightarrow$ Adolescent alcohol use & $-.196^{* *}$ & -.104 \\
Conscientiousness $\rightarrow$ Adolescent alcohol use & -.125 & $-.175^{*}$ \\
Total effects & & .099 \\
$\begin{array}{l}\text { Extraversion } \rightarrow \text { Peers' alcohol use at the } \\
\text { weekend }\end{array}$ & $.287^{* *}$ & -.122 \\
Agreeableness $\rightarrow$ Peers' alcohol use at the & $-.275^{* *}$ & $-.205^{*}$ \\
weekend & & $.170^{\dagger}$ \\
Conscientiousness $\rightarrow$ Peers' alcohol use at the & $-.176^{\dagger}$ & $-.220^{*}$ \\
weekend & $.269^{\dagger}$ & $-.178^{*}$ \\
Extraversion $\rightarrow$ Adolescent alcohol use & $-.490^{* *}$ & $.855^{* * *}$ \\
Agreeableness $\rightarrow$ Adolescent alcohol use & -.166 & \\
Conscientiousness $\rightarrow$ Adolescent alcohol use & & \\
Peers' alcohol use at the weekend $\rightarrow$ & $.713^{* * *}$ & \\
Adolescent alcohol use & &
\end{tabular}

${ }^{\dagger} p<.10 .{ }^{*} p<.05 .{ }^{* *} p<.01 .{ }^{* * *} p<.001$. 


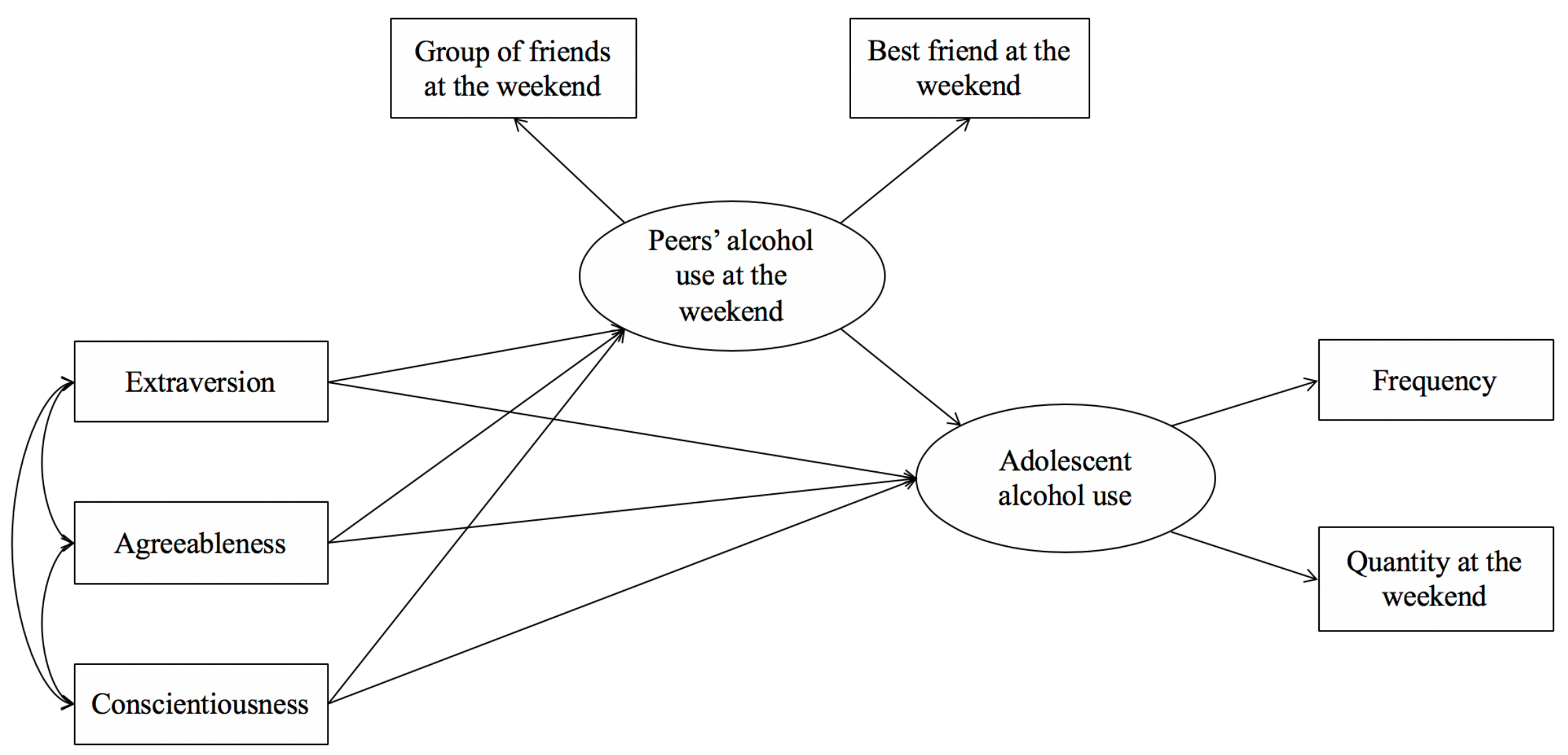

Figure 1. Hypothesized Model. 


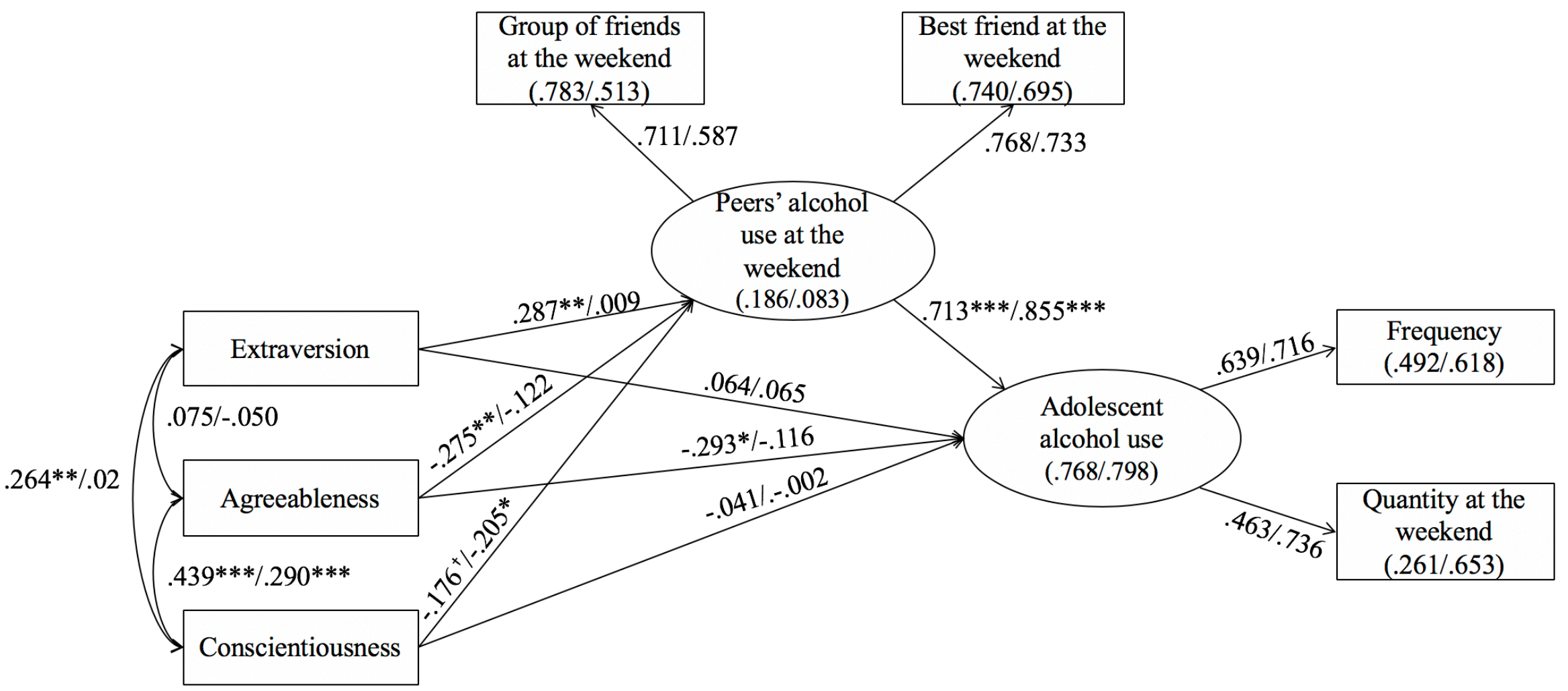

Figure 2. Independent structural equation models for Scottish $(n=120)$ and Spanish $(n=221)$ adolescents shown in one single figure. Correlations among personality dimensions, factor loadings for peers' and own alcohol use and standardized solutions before the slash are for Scottish adolescents; those after the slash are for Spanish adolescents. The effect of age and gender was controlled for by covarying both variables with all the observed variables. Between brackets are the $R^{2}$ (Scottish sample before the slash and Spanish sample after the slash). ${ }^{\dagger} p<.10 .{ }^{*} p<.05 .{ }^{* *} p<.01 .{ }^{* * *} p<.001$. 